\title{
Photopolarimetric monitoring of OJ 287 in 1994-1997
}

\author{
Yu. S. Efimov ${ }^{1}$, N. M. Shakhovskoy ${ }^{1}$, L. O. Takalo ${ }^{2}$, and A. Sillanpää² \\ 1 Crimean Astrophysical Observatory and Isaac Newton Institute of Chile, Crimean Branch, Ukraine \\ e-mail: efimov@astro.crao.crimea.ua \\ 2 Tuorla Observatory, Tuorla, 21500 Piikkiö, Finland \\ e-mail: takalo@astro.utu.fi
}

Received 11 December 2000 / Accepted 19 October 2001

\begin{abstract}
We present and discuss the $U B V R I$ photopolarimetric data of blazar OJ 287 obtained at the Crimean Astrophysical Observatory from January 1994 until April 1997 as a part of the international OJ-94 campaign. The variations of brightness and linear polarization detected in all used optical bands are similar. No clear correlation was found between the brightness variations and polarization parameters. The most prominent characteristic in the object variability is the apparent continuous rotation of the observed polarization position angle in 19941996. The rate of this rotation over two observational seasons in 1994-1995 and 1995-1996 is about 4.92/day in a clockwise direction. This rate corresponds to the full $360^{\circ}$ rotation of the polarization plane in about 73 days. We consider the rotation of the plane of polarization as direct evidence for helical magnetic field structure in the jet of OJ 287.
\end{abstract}

Key words. BL Lacertae objects: individual: OJ 287 - polarization

\section{Introduction}

After the optical identification of OJ 287 by Blake in 1970 (Blake 1970), three large optical outbursts have been identified in OJ 287: in 1972-1973, in 1982-1983 and in 1994-1996. It is the only blazar with strict periodicity of 11.65 years between the large outbursts of amplitude $\approx 5.5^{\mathrm{m}}$ in the $V$-band (Sillanpää et al. 1988; Sillanpää et al. 1996). A number of polarimetric observations were made during the first two outbursts (Kikuchi et al. 1976; Hagen-Thorn 1980; Kikuchi 1989; Takalo 1994 and references therein).

According to the binary black hole model by Sillanpää et al. (1988) and Lehto \& Valtonen (1996), next outbursts should have occured in 1994 and 1996, with an eclipse expected in 1998.

In 1993 a large international campaign called "OJ-94 project" was initiated. The main aim of this project was a long-term study of the behaviour of some bright blazars using simultaneous photometric and polarimetric observations across the electromagnetic spectrum. Such observations contain unique information on the physical parameters of the jets in blazars and the topology of their magnetic field. The main target of the monitoring program was blazar OJ 287. The plan was to verify the predicted outbursts.

Send offprint requests to: L. O. Takalo,

e-mail: takalo@astro.utu.fi
Previously many authors (e.g. Shakhovskoy \& Efimov 1977; Hagen-Thorn 1981; Kikuchi 1988; Sillanpää et al. 1991; Sillanpää et al. 1992) have discussed the behaviour of the optical polarization of OJ 287. However, the numerous previous polarization observations were usually obtained during rather short time intervals and only few simultaneous multi-wavelength observations were made.

The behaviour of the polarization parameters and brightness of OJ 287 in 1993-1998 across the spectrum from the gamma-ray to the radio domain is discussed in Pursimo et al. (2000). It is seen from their Figs. 4 and 5 that in the whole optical domain the degree of polarization $P$ is mostly scattered around $10 \%$, ranging from $0 \%$ to $20 \%$ with a maximum about $30 \%$ with the brightness' level being from 2 to $4 \mathrm{mJy}$ in the $V$-band, and the position angle PA sweeping between $0^{\circ}-180^{\circ}$. The degree of the polarization is close to the average value of about $10 \%$ during the outbursts and rose above $20 \%$ at a moderate brightness level. No obvious correlation between the polarization parameters and the brightness is found.

Results of our photopolarimetry, obtained in the course of OJ-94 project, were partly presented in the meetings at Orilampi, Finland in 1994 (Efimov \& Shakhovskoy 1994), in Oxford, England in 1995 (Efimov \& Shakhovskoy 1996), in Girona, Spain in 1996, and in Perugia, Italy in 1997 (Efimov \& Shakhovskoy 1998a).

In this paper we present a more detailed analysis of optical polarimetric data of OJ 287 obtained during 82 nights from January 12, 1994 till April 30, 1997. 
The data can be obtained from the OJ-94 project archive (http://www.astro.utu.fi/oj94).

The outline of the paper is as follows: in Sect. 2 we describe the observation and data reduction procedure. Section 3 contains the analysis of the general behaviour of the brightness, color, spectral index and polarization parameters. The variations of the position angle are considered in Sect. 4 and discussed in Sect. 5. In Sect. 6 we present a summary of our main results.

\section{Observations and data reduction}

All our observations were carried out with the $125 \mathrm{~cm}$ telescope at the Crimean Astrophysical Observatory (Ukraine), using the computer controlled $U B V R I$ Double Image Chopping Photopolarimeter, developed at the Helsinki University Observatory by V. Piirola (Piirola 1973, 1988; Korhonen et al. 1984). This device has been in operation at the Crimean Astrophysical Observatory since May 1981.

The instrument has two operational modes: photometric mode with elimination of the background close to the object, and two polarimetric modes to measure linear and/or circular polarization. In these modes the background is measured before and after a set of the observations of the object. An important advantage of this device is the automatic elimination of the sky light polarization.

In both polarimetric modes the instrument provides measurements of the intensity and polarization simultaneously in $U B V R I$ bands using four dichroic filters which split the light into the five photomultipliers, whose spectral regions are centered at $0.36,0.44,0.53,0.69$, and $0.83 \mu \mathrm{m}$, respectively. The passbands are close to the standard $U B V$ (Johnson) and $R I$ (Cousins) systems, allowing an accurate conversion of the photometric data from the instrumental to the standard system.

The observations have been carried out in the linear polarization measurement mode. In the course of polarimetric measurements the instrument retarder plate is rotated with 22.5 steps in front of a polarizer. One measurement consists of eight integrations in different orientations of the retarder. With the typically used integration time of $10 \mathrm{~s}$ in polarimetric mode (for both the ordinary and extraordinary beams) one full measurement takes $3.06 \mathrm{~min}$. The number of measurements varied from 4 to 44 depending on the sky conditions. Since the number of individual brightness estimations is rather large, the uncertainty in the brightness estimation of OJ 287 is usually $<0 .{ }^{\mathrm{m}} 02$.

The observer can control the process of the measurements by the on-line listing of the individual and average brightness and normalized polarization Stokes parameters with their rms errors in the instrumental system in all bands without interrupting the observations.

The instrumental polarization in each color, determined from the observations of unpolarized standard stars, is usually very small $(<0.2 \%)$. The calibration of the position angle was made observing stars with well

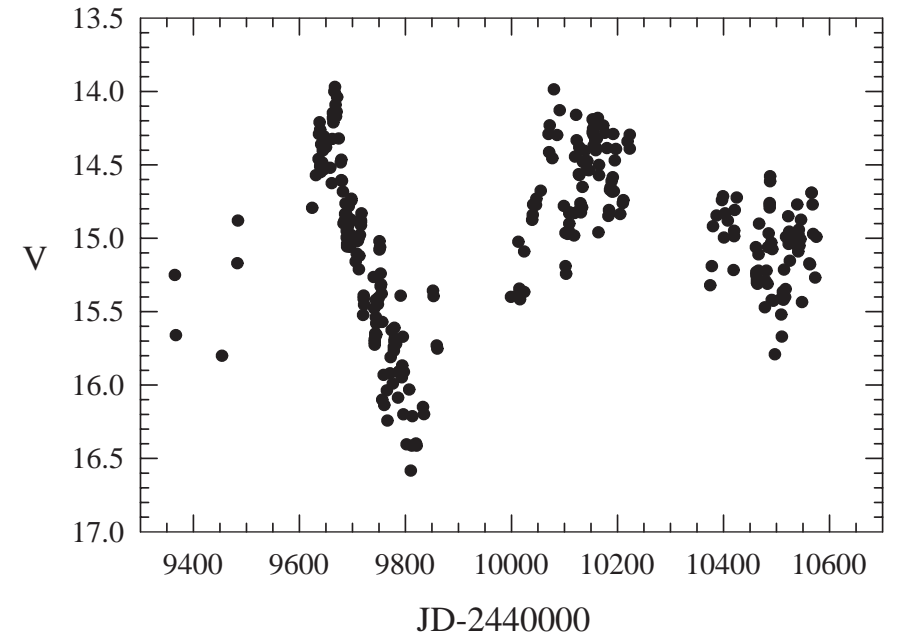

Fig. 1. $V$-band light curve of OJ 287 observed during the years 1994-1997 in the frame of the OJ-94 project at the Crimean Astrophysical Observatory.

known large interstellar polarization (Serkowski 1974; Hsu $\&$ Breger 1982). Star 2 from Smith et al. (1985) was used as the photometric reference star.

Before reduction all raw data were checked to eliminate evident bad points from the observations. The data reduction was performed using the software package developed by V. Piirola, which performs all the required corrections (background subtraction, correction for the instrumental polarization, transformation into the equatorial system, transformation from instrumental magnitudes to the standard system, calculation of the nightly weighted means of the normalized Stokes parameters $P_{x}=P \cos (2 \mathrm{PA})$, $P_{y}=P \sin (2 \mathrm{PA})$, where $P$ is the degree of polarization, and $\mathrm{PA}$ is the position angle of polarization plane in the equatorial system. Data obtained after the first run of the programs are checked again to correct the initial files for doubtful measurements (cirrus, malfunction of the equipment, etc.) and then a second run of the programs is performed to get final results. All uncertain data were deleted from the data presented below.

\section{General behaviour}

\subsection{Brightness variations}

Our photopolarimetric observations of OJ 287 were taken in 1994-1997. During this time there were two outbursts in OJ 287: one sharp outburst in October/November 1994 (JD 2449600-2449750) and a more prolonged outburst, with large brightness variability in November 1995May 1996 (JD 2450000-2450200), divided by a deep minimum during spring 1995 (JD 2449750-2449850). The $V$-band brightness variations of OJ 287 in this time interval were between $16^{\mathrm{m}} \cdot 7$ and $13^{\mathrm{m}} \cdot 7$. The light curve of OJ 287 in $V$-band, based on the observations at the Crimean Astrophysical Observatory (see the archive in the WEB site (http://www.astro.utu.fi/oj94)), is shown in Fig. 1. 


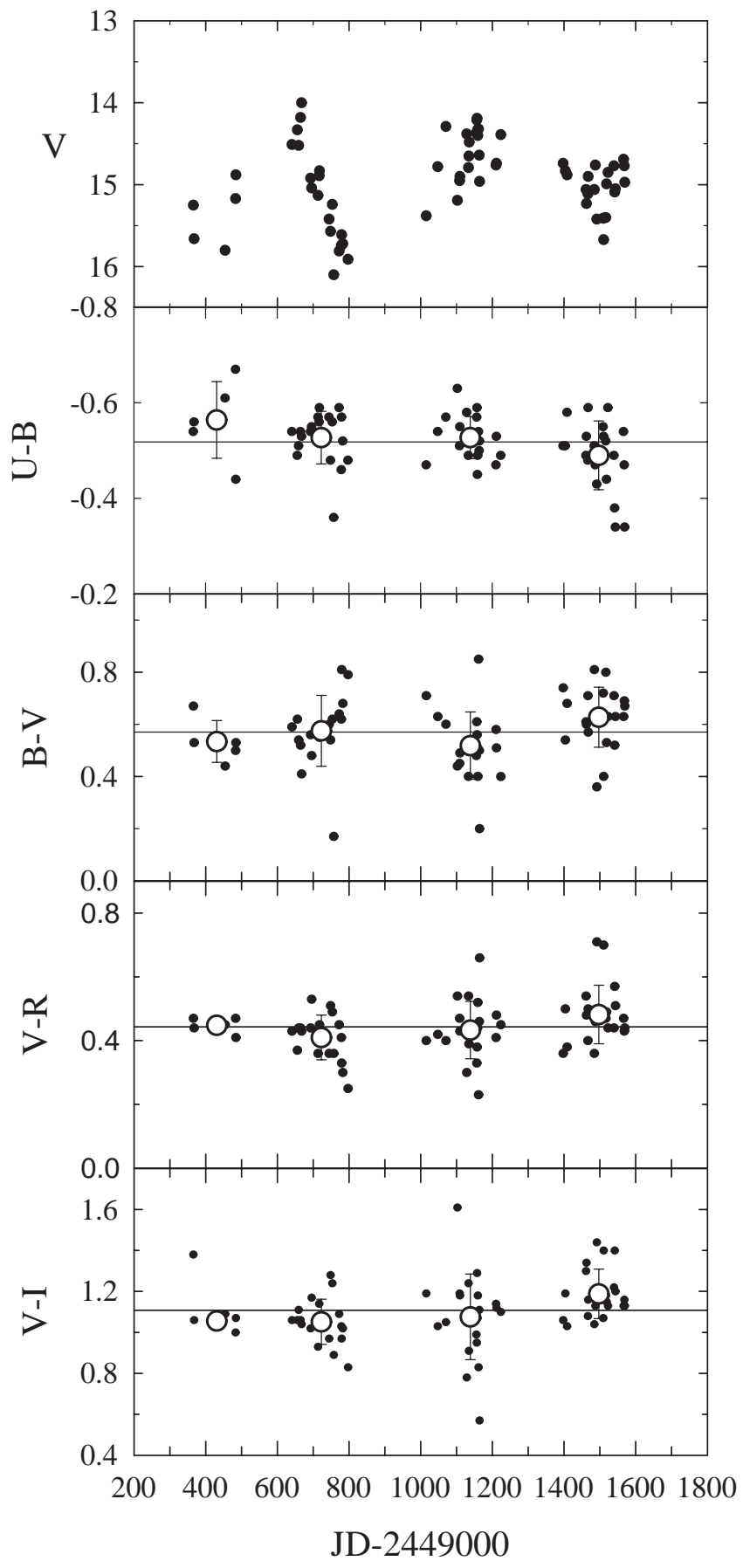

Fig. 2. Variation of the colour indices $U-B, B-V, V-R$ and $V-I$ of OJ 287 observed during the years 1994-1997 in the frame of the OJ-94 project. The upper panel shows the brightness of OJ 287. The solid lines correspond to the average color indices during 1994-1997. The open circles with bars represent the average data and the rms scattering of the data for each season. Here and in all other figures the used data are from the Crimean Astrophysical Observatory.

According to Pursimo et al. (2000, Fig. 2), there are several components in the brightness variations: 1) large outbursts with an amplitude up to $3^{\mathrm{m}}$, lasting 200-300 days; 2) small light variations with amplitudes up to of about $1^{\mathrm{m}}$, lasting several days, 3) microvariability
Table 1. Comparison of the average colour indices of OJ 287 in 1994-1997.

\begin{tabular}{lccccr}
\hline Season & $U-B$ & $B-V$ & $V-R$ & $V-I$ & $N$ \\
\hline $1993 / 1994$ & $-0.56 \pm 0.08$ & $0.53 \pm 0.08$ & $0.45 \pm 0.02$ & $1.06 \pm 0.03$ & 5 \\
$1994 / 1995$ & $-0.53 \pm 0.06$ & $0.58 \pm 0.14$ & $0.41 \pm 0.07$ & $1.05 \pm 0.11$ & 19 \\
$1995 / 1996$ & $-0.53 \pm 0.04$ & $0.52 \pm 0.13$ & $0.43 \pm 0.09$ & $1.08 \pm 0.21$ & 21 \\
$1996 / 1997$ & $-0.49 \pm 0.07$ & $0.63 \pm 0.11$ & $0.48 \pm 0.09$ & $1.19 \pm 0.12$ & 21 \\
global & $-0.51 \pm 0.06$ & $0.57 \pm 0.13$ & $0.44 \pm 0.09$ & $1.11 \pm 0.16$ & 66 \\
\hline
\end{tabular}

with time scale from tens of minutes to hours and with amplitudes up to $0 .{ }^{\mathrm{m}} 3 /$ hour (see also Dultzin-Hacyan et al. 1997; Jia et al. 1998). The variability behaviour is very similar in all optical bands. Hence, it appears that no color variations occurred in this time interval.

\subsection{Color variations}

The claimed correlation between the color variation and brightness level is controversial. In the study of the longterm optical and spectral index variability of OJ 287 between 1972-1988, covering the great outburst in 1983 and two minima (in 1976 and in 1984-1988), Takalo \& Sillanpää (1989) found clear dependencies of the $B-V$ color index on time and on the brightness of OJ 287. The objects' behaviour was studied at the same time by Carini et al. (1992). According to Takalo \& Sillanpää and Carini et al., OJ 287 became slightly redder when fainter. The scatter in the $B-V$ index increased when the object became fainter than $14^{\mathrm{m}} 5$.

A close correlation between the $B-V$ color index and the $V$ magnitude was found by Fan et al. (1998) based on a dozen points obtained in winter 1994-95, at the falling branch of brightness after the maximum in 1994 .

However, no dependence between the $B-V$ color and the $V$-band brightness was found during the maximum brightness in 1972 (Frölich et al. 1974) and in 1983 (Moles et al. 1984), or during the minimum in 1974 (Rieke \& Kinman 1974). Some authors have pointed out that at deep minimum the object becomes slightly redder due to the contribution from the host galaxy. This would be noticeable when the object's brightness falls close to the extremely faint value $B \approx 17$ (Lyuty 1976). The variation of color indices $U-B, B-V, V-R$ and $V-I$ of OJ 287 observed during the years 1994-1997 are shown in Fig. 2 (filled circles). The open circles correspond to the values of color indices averaged over each season and the solid lines mark the overall color indices. The error bars are not shown since they are smaller than the symbol size. The upper panel shows the brightness of OJ 287. As it can be seen, the scattering is large. To study the behaviour of color indices during all four seasons we compared the average color indices in every season to the overall average (Table 1). Table 1 and Fig. 2 show that in spite of the large change in the OJ 287 brightness between 1994-1997, no systematic color variations but only random fluctuations $U-B, B-V, V-R, V-I$ were found in our observations. 
Table 2. Spectral index summary from literature.

\begin{tabular}{lcc}
\hline Time interval & $\alpha$ (optics) & Ref Comments \\
\hline 1971 Oct. $24 \rightarrow$ Oct. 26 & 1.40 & 1,19 max \\
1972 Sept. $\rightarrow$ Oct. & 1.0 & 2 outburst \\
1972 Jan. $\rightarrow$ Mar. & 0.9 & 3 outburst \\
1972 Jan. & 1.248 & 4 outburst \\
1974 Jan. & 1.2 & 5 outburst \\
1973 Jan. $\rightarrow 1975$ Mar. & $0.4 \rightarrow 1.0$ & 6,19 min \\
1971 Dec. $\rightarrow 1976$ Apr. & $0.7 \rightarrow 3.0$ & 7,19 max, min \\
1980 Oct. 24 & $1.44 \rightarrow 1.63$ & 8 moderate \\
1980 Oct. $23 \rightarrow 1981$ May 11 & 1.22 & 9 moderate \\
1981 Dec. & 1.20 & 10 faint \\
1982 Nov. $\rightarrow 1984$ Jun. & $1.25 \rightarrow 1.36$ & 11 outburst \\
1983 Jan. $7 \rightarrow$ Jan. 10 & 1.25 & 20 outburst \\
1983 Feb. $3 \rightarrow$ Mar. 9 & $1.18 \rightarrow 1.36$ & 12 outburst \\
1983 Feb. $15 \rightarrow$ Feb. 18 & $0.7 \pm 0.15$ & 13 outburst \\
1984 Nov. $\rightarrow 1991$ Feb. & $1.18 \rightarrow 1.61$ & 14 faint \\
1985 Mar. $22 \rightarrow$ Nov. 8 & $1.4 \rightarrow 2.0$ & 15,19 min \\
$1971 \rightarrow 1988$ & $0.7 \rightarrow 2.1$ var & 16 min, max \\
$1993 \rightarrow 1995$ & $1.52 \rightarrow 1.55$ & 17 min, max \\
1994 Jan. $14 \rightarrow 21$ & $1.46 \rightarrow 1.65$ & 22 min \\
$1994 \rightarrow 1996$ & $1.73 \rightarrow 1.96$ & 18 min, max \\
1994 Jan. $\rightarrow 1997$ Apr. & $0.5 \rightarrow 2.0$ & 21 max, min \\
\hline
\end{tabular}

References:

1 Gilles, A., et al. 1972, C. R. Acad. Sci., Ser. B, 274, 201

2 Dyck, H. M. 1971, N. Phys. Sci., 234, 71

3 Kinman, T. D., et al. 1974, AJ, 79, 349

4 Visvanathan, N. 1973, ApJ, 185, 145

5 Rieke, G. H. \& Kinman, T. D. 1974, ApJ, 192, L115

6 Kikuchi, S., et al. 1976, PASJ, 28, 117

7 Lyuty, V. M. 1976, Peremennye Zvezdy, 20, 243

8 Worrall, D. M., et al. 1982, ApJ, 261, 403

9 Sitko, M. L., et al. 1983, PASP, 95, 724

10 Landau, E., et al. 1983, ApJ, 268, 68

11 Smith, P. S., et al. 1987, ApJSS, 64, 459

12 Moles, M., et al. 1984, MNRAS, 211, 621

13 Maraschi, L., et al. 1983, A\&A, 127, L17

14 Falomo, R. et al. 1994, ApJSS, 93, 125

15 Sitko, M. L., \& Sitko, A.K. 1991, PASP, 103, 160

16 Takalo, L. O., \& Sillanpää, A. 1989, A\&A, 218, 45

17 Hagen-Thorn, V. A., et al. 1998, A\&AS, 133, 353

18 Marchenko, S. G., et al. 1998, OJ-94 annual meeting 1997,

Multifrequency monitoring of Blazars, ed. Tosti G. and

Takalo, L., Perugia University Observatory Publications, 3, 36

19 Matthews, T. A., \& Sandage, A.R. 1963, ApJ, 138, 30

20 Holmes, P. A., et al. 1984b, MNRAS, 211, 497

21 This paper

22 Dultzin-Hacyan, D., et al. 1997, Rev. Mex. Astron. Astrofís., 33, 17

\subsection{Spectral index variations}

It is well known that the jet radiation in blazars has a synchrotron nature and a power-law energy distribution that follows $\nu^{-\alpha}$ (at least over limited frequency ranges), where $\alpha$ is the spectral index.

The data on the spectral index variations are also controversial. The obvious evidence for longterm spectral index variations in $1972-1988$ was found
Table 3. Spectral index summary from our observations in 1994-1997.

\begin{tabular}{|c|c|c|c|c|c|c|c|}
\hline Date & JD-2449000 & $\alpha$ & $\sigma$ & Date & JD-2449000 & $\alpha$ & $\sigma$ \\
\hline 940112 & 365.585 & 1.95 & 0.17 & 960221 & 1135.452 & 1.45 & 0.11 \\
\hline 940114 & 367.558 & 1.48 & 0.10 & 960312 & 1155.401 & 1.38 & 0.10 \\
\hline 940411 & 454.340 & 1.35 & 0.09 & 960313 & 1156.366 & 1.43 & 0.15 \\
\hline 940510 & 483.308 & 1.31 & 0.01 & 960314 & 1157.408 & 1.65 & 0.20 \\
\hline 940511 & 484.321 & 1.57 & 0.15 & 960315 & 1158.367 & 1.55 & 0.13 \\
\hline 941014 & 640.587 & 1.56 & 0.11 & 960316 & 1159.348 & 1.50 & 0.10 \\
\hline 941029 & 655.612 & 1.62 & 0.16 & 960318 & 1161.428 & 1.62 & 0.26 \\
\hline 941102 & 659.589 & 1.57 & 0.12 & 960320 & 1163.397 & 1.55 & 0.11 \\
\hline 941107 & 664.568 & 1.48 & 0.10 & 960506 & 1210.298 & 1.66 & 0.15 \\
\hline 941110 & 667.587 & 1.33 & 0.10 & 960507 & 121 & 1.54 & 0.09 \\
\hline 941205 & 692.626 & 1.50 & 0.10 & 960519 & 1223.318 & 1.40 & 0.12 \\
\hline 941208 & 695.600 & 1.55 & 0.07 & 961109 & 1397.590 & 1.75 & 0.18 \\
\hline 941226 & 713.470 & 1.40 & 0.14 & 961115 & 1403.583 & 1.66 & 0.10 \\
\hline 941229 & 716.541 & 1.62 & 0.12 & 961120 & 1408.586 & 1.60 & 0.15 \\
\hline 941230 & 717.519 & 1.49 & 0.09 & 970112 & 1461.438 & 1.88 & 0.10 \\
\hline 950126 & 744.389 & 1.45 & 0.14 & 970113 & 1462.544 & 1.84 & 0.15 \\
\hline 950130 & 748.423 & 1.76 & 0.12 & 970117 & 1466.552 & 1.77 & 0.16 \\
\hline 950204 & 753.406 & 1.76 & 0.11 & 970118 & 1467.555 & 1.61 & 0.08 \\
\hline 950223 & 772.370 & 1.62 & 0.11 & 970204 & 1484.399 & 1.82 & 0.20 \\
\hline 950301 & 778.354 & 1.64 & 0.15 & 970207 & 1487.435 & 1.72 & 0.12 \\
\hline 950302 & 779.356 & 1.70 & 0.20 & 970211 & 1491.377 & 1.80 & 0.13 \\
\hline 950305 & 782.418 & 1.60 & 0.19 & 970301 & 1509.390 & 1.75 & 0.13 \\
\hline 950320 & 797.302 & 1.60 & 0.25 & 970302 & 1510.368 & 1.73 & 0.10 \\
\hline 951024 & 1015.584 & 1.87 & 0.17 & 970308 & 1516.440 & 1.96 & 0.15 \\
\hline 951125 & 1047.571 & 1.59 & 0.13 & 970310 & 1518.370 & 1.67 & 0.12 \\
\hline 951218 & 1070.456 & 1.53 & 0.12 & 970314 & 1522.447 & 1.64 & 0.12 \\
\hline 960119 & 1102.411 & 1.80 & 0.28 & 970331 & 1539.300 & 1.89 & 0.15 \\
\hline 960125 & 1108.485 & 1.54 & 0.12 & 970402 & 1541.295 & 1.95 & 0.14 \\
\hline 960126 & 1109.516 & 1.53 & 0.13 & 970404 & 1543.277 & 1.93 & 0.15 \\
\hline 960214 & 1128.339 & 1.17 & 0.15 & 970427 & 1566.302 & 1.69 & 0.11 \\
\hline 960219 & 1133.461 & 1.56 & 0.11 & 970429 & 1568.302 & 1.83 & 0.15 \\
\hline 960220 & 1134.447 & 1.35 & 0.13 & 970430 & 1569.302 & 1.89 & 0.18 \\
\hline
\end{tabular}

by Takalo \& Sillanpää (1989), when the spectral index changed from 0.7 in 1972 to 2.1 in 1988 . The spectrum became steeper when OJ 287 faded in $U B V R I$ (Moles et al. 1984) and in the near $I R$ during the dimming phase after the strong outburst in 1983 (Gear 1986). On the other hand, many authors observing OJ 287 from 1972 to 1988 noted that the spectral index $\alpha$ usually exhibits small and random variations, independent of the objects brightness level. Subsequent studies of OJ 287 didn't reveal any regular changes in the spectral index after 1988 (Table 2). During this time the typical value for the spectral index indicated a steeper spectrum in comparison with the one in the beginning of the seventies.

The multi-band observations during 1994-1997 cover the two outbursts of OJ 287 and allow us to study the behaviour of the spectral index $\alpha$ at different levels of the brightness. The $U B V R I$ values for every date were converted to fluxes in mJy using the calibration by O'Dell (1978) and fitted by a power law $\left(F_{\nu} \propto \nu^{-\alpha}\right)$. The correlation coefficients of the fits are all $>0.95$ for all cases. The values of obtained spectral indices $\alpha$ and its standard error $\sigma$ are given in Table 3 . 


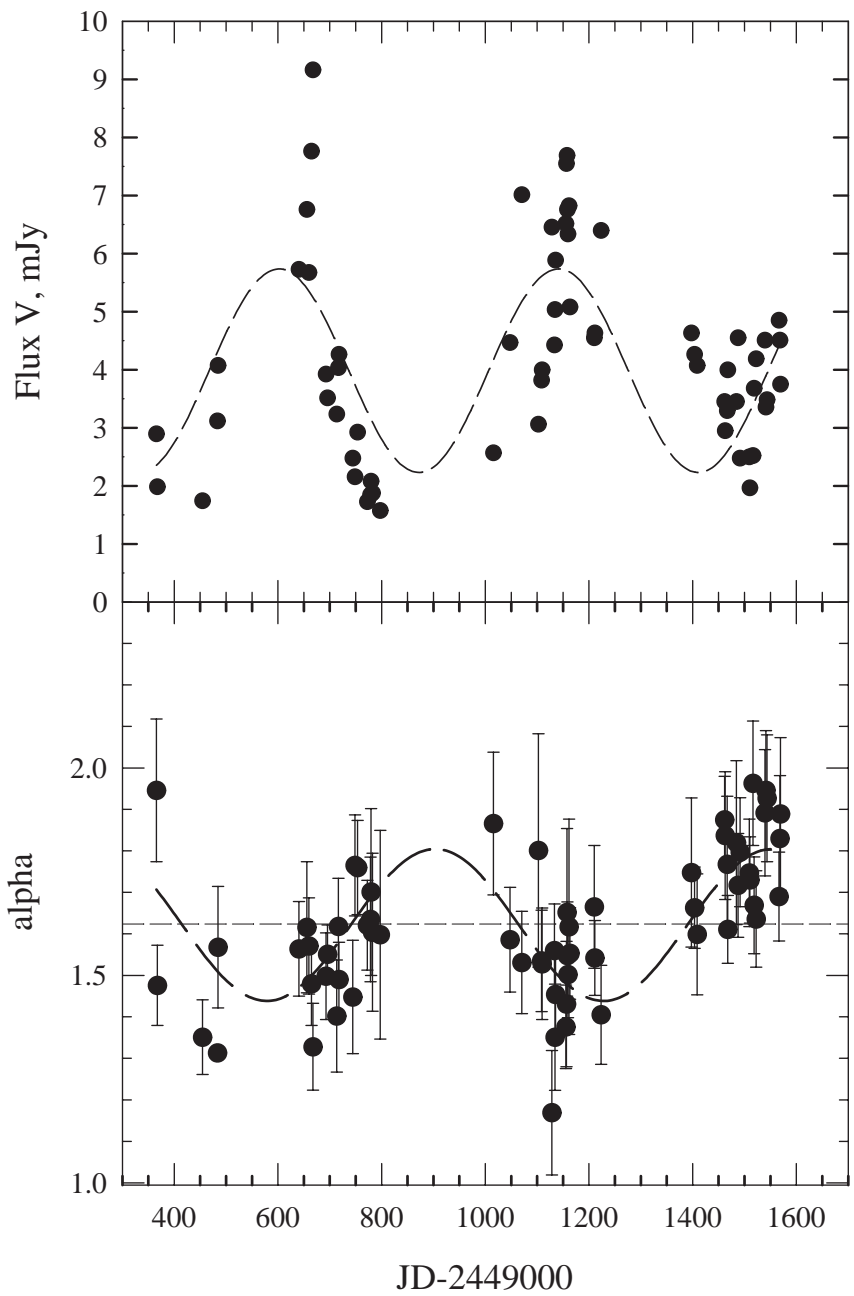

Fig. 3. Comparison of variations of the spectral index $\alpha$ of OJ 287 (bottom panel) and its brightness of OJ 287 in $V$ band in mJy (upper panel) with time in 1994-1997. The wave-shaped dashed lines in both panels show the approximation of observed variations of the brightness and spectral index by the sinusoidal function $y=y_{0}+a * \sin (2 \pi / b+c)$, where $y_{0}=3.981 \pm 0.200$, $a=1.753 \pm 0.261, b=538.995 \pm 17.750^{\mathrm{d}}, c=0.832 \pm 0.418 \mathrm{rad}$ for spectral index and $y_{0}=1.621 \pm 0.183, a=0.183 \pm 0.028$, $b=649.374 \pm 27.563^{\mathrm{d}}, c=5.400 \pm 0.390 \mathrm{rad}$ for brightness. The straight dashed line in the bottom panel is the average level of $\alpha=1.62 \pm 0.18$. The bars show the rms errors of $\alpha$.

The behaviour of the spectral index is shown in Fig. 3 and reveals a large scatter of the spectral index, from 1.17 (JD 2441128, at the rising branch of the second outburst) to 1.96 (JD 2450516, at the beginning of the falling branch of the second outburst) with the average value $1.62 \pm 0.18$ (the thin strict dashed line).

To the modelling of the lacertids it is important to know if the spectral energy distribution depends on the brightness of the object. The spectral energy distribution of OJ 287 shows a tendency to be flatter when the object is at a high brightness level (the thick wave-shaped dashed line in Fig. 3). A clearer relation between the spectral index and brightness is apparent in Fig. 4.

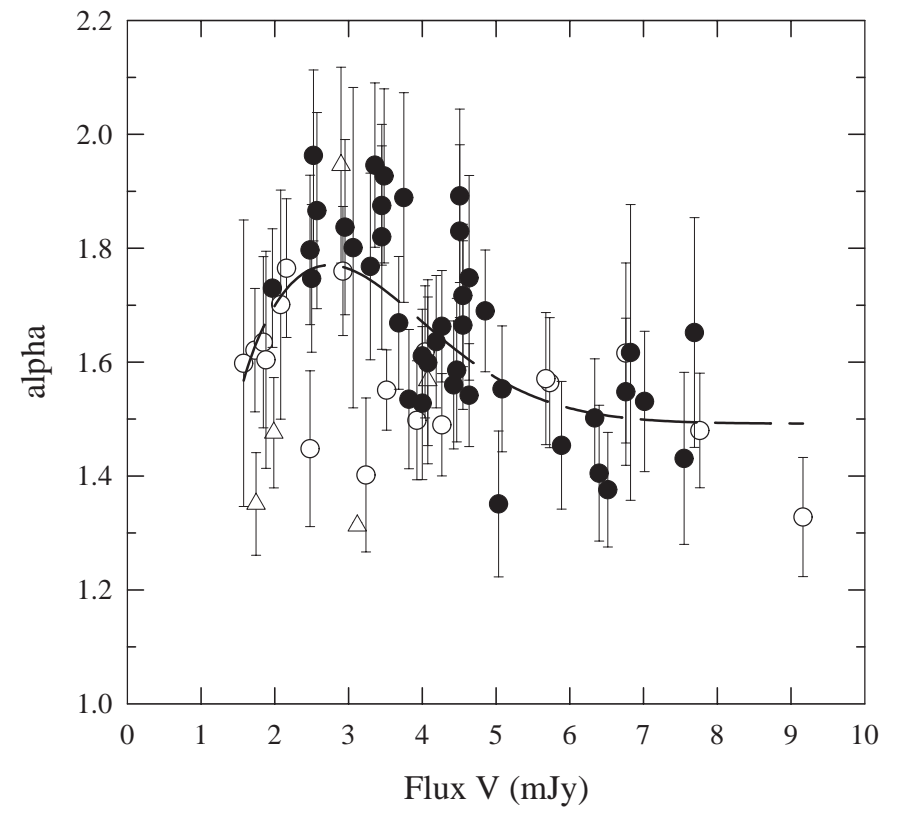

Fig. 4. Correlation between $V$-band (mJy) brightness variations of OJ 287 and the spectral index $\alpha$. The data for different time intervals are shown separately for 19941995 (JD 2449315-2449484, open triangles), for 1995-1996 (JD 2449640-2449797, open circles) and for 1996-1997 (JD 2450015-24500569, filled circles). The dashed line represents the general behaviour of the spectral index $\alpha$ with variation of the flux in the $V$-band.

This demonstrates that the spectral energy distribution is steepest in the region of 3-4 mJy. The relation was most evident during the second outburst of OJ 287 in 1996-1997. Examples of the spectral energy distribution of OJ 287 at the maximum and minimum brightness are displayed in Fig. 5.

\subsection{Comparison of the variations of polarization and brightness with time}

There are several blazars with observed correlation between the degree of polarization and brightness in optical region: (PKS 0735+178, Shakhovskoy \& Efimov 1977; 3C 345, Smith et al. 1986; PKS 0215+015, Kikuchi et al. 1989; Mkn 421, Tosti et al. 1998; 3C 66A, Efimov \& Shakhovskoy 1998a; ON 231, Efimov \& Shakhovskoy 1998b). This relation between polarization and brightness variations was recently studied by Fan et al. (2000) and explained in the frame of a beaming model for blazars.

In addition, there are some cases where such a correlation is absent or doubtful (BL Lac, Sillanpää et al. 1993; PKS 0109+224, Valtaoja et al. 1993; S5 0714+714, Kümmel et al. 1994).

It seems that in all the above cases the relation between polarization and brightness (when seen) occured only at the time of outbursts. In the case of OJ 287, the only well-studied object, this relation (see below) appears as a broad maximum near moderate, not the maximum, brightness level. 


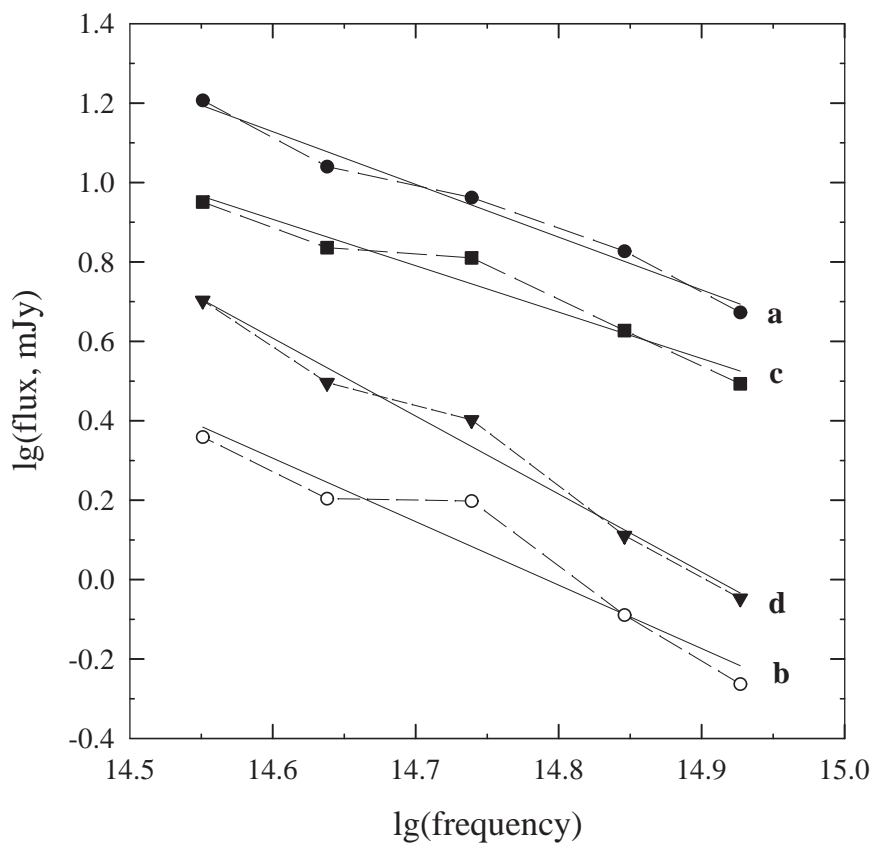

Fig. 5. Spectral energy distribution of OJ 287 from our observations. The data points are connected with the dotted and dashed lines. The solid straight lines are the best fits to the data. The letters at the lines indicate the JD: a) filled circles, JD 2449667, $\max , \alpha=1.33 \pm 0.10$; b) open circles, JD $2449797, \min , \alpha=1.60 \pm 0.17$; c) filled squares, JD $2450128, \max , \alpha=1.17 \pm 0.15$; d) open squares, JD 2450516 , min, $\alpha=1.96 \pm 0.15$.

As one can see from the comparison of the polarization variability in various passbands (Pursimo et al. 2000), the general behaviour of the polarization $P$ and the position angle PA in 1994-1997 was very similar in all bands. An example of the general behaviour of our polarimetric data in $R$-band obtained from January 12, 1994 till April 30, 1997 is shown in Fig. 6.

We mark several most imposing events in Fig. 6:

(1) large night-to-night scatter in the degree of polarization around the systematic slow increase of $P$ with time from few per cents in 1994 to 28\% in 1997 (JD 2450550);

(2) progressive increase of the average degree of polarization with time at a rate of $0.012 \%$ /day (the dashed straight line in the middle panel);

(3) the increase of the polarization degree from $10 \%$ to $28 \%$ in 1996-97 (near JD 2450520);

(4) the continuous rotation of the position angle from $210^{\circ}$ to $30^{\circ}$ during the fading of the object in 1994-95 (near JD 2449760);

(5) the same sense of rotation of the polarization angle in 1995-96 (near JD 2450120) during the bright stage of OJ 287.

The PA behaviour will be considered in detail later.

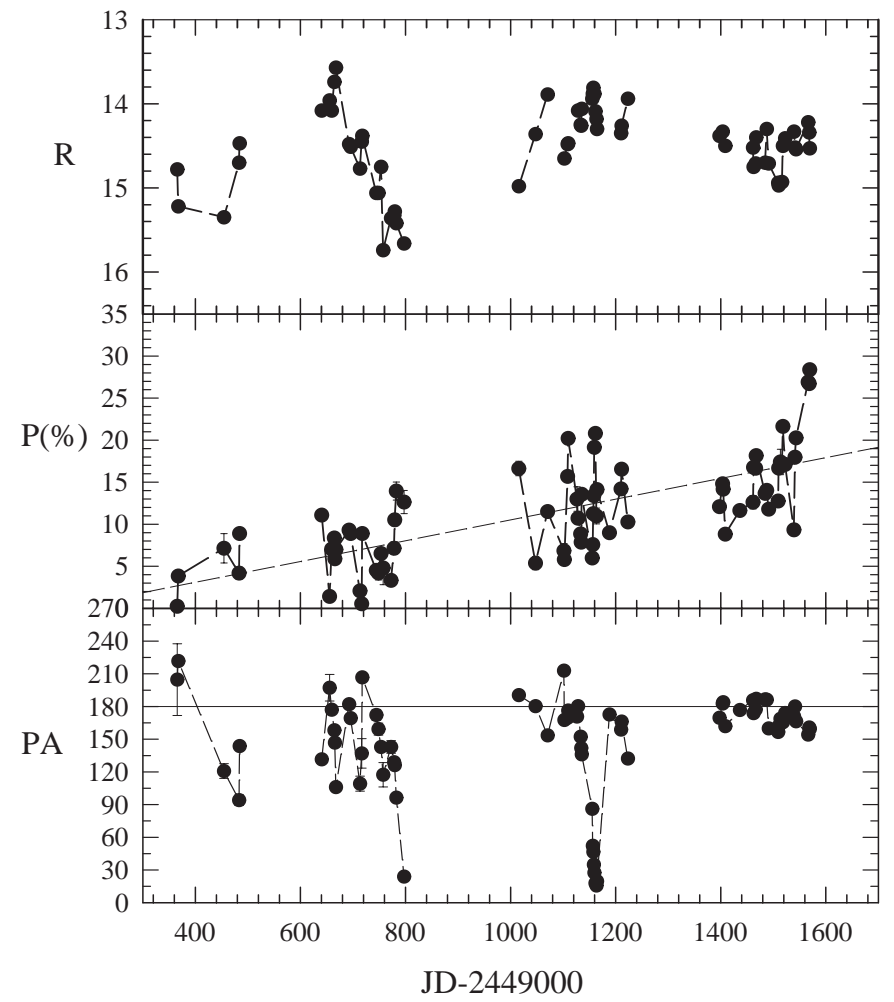

Fig. 6. Photometric and polarimetric behaviour of OJ 287 in the $R$-band with time in JD. The variations of the observed brightness (upper panel), degree of polarization $P(\%)$ (middle panel) and position angle PA (bottom panel) of OJ 287 in the $R$ band during the monitoring campaign are shown (see text). The bars are the rms observational errors. The dashed lines connect the successive points inside each season of observations. The thin straight line on the bottom panel marks the $180^{\circ}$ level. Due to an ambiguity of $180^{\circ}$ some points on the bottom panel located close to $0^{\circ}$ are shifted up to $180^{\circ}$.

\subsection{Variation of the polarization parameters with brightness}

The photopolarimetric data of OJ 287 give a unique possibility to check the dependence of the polarization parameters $P$ and PA on brightness level based on simultaneous observations over a three year period. The comparison is made in Fig. 7 where filled circles represent individual data and open circles show the mean values of $P$ and PA averaged over the Stokes parameters $P_{x}, P_{y}$ binned on $0 .{ }^{\mathrm{m}} 5$ intervals. Figure 7 shows that the degree of polarization reaches its maximum at the moderate brightness of about $14 .^{\mathrm{m}} 5$. The position angle remains close to $0^{\circ}$ over a wide range of brightness levels.

\subsection{Comparison of the polarization variations with spectral index}

It is well known that the degree of linear polarization for the synchrotron emission depends on the spectral index $\alpha$, on the electron distribution and on the magnetic field structure. 


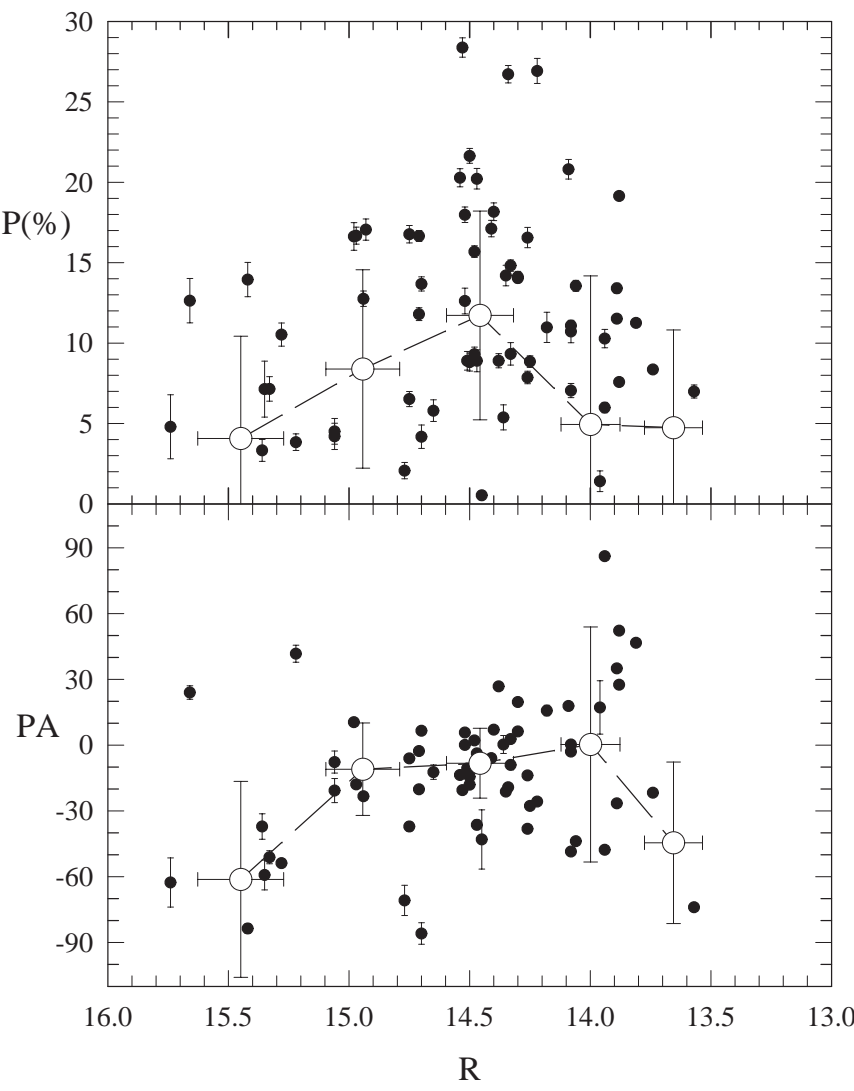

Fig. 7. Comparison of the variations of the polarization degree $P$ (upper panel) and the position angle PA (bottom panel) of OJ 287 with the variations of its brightness in $R$-band. The filled circles represent the individual data, and open circles show the mean values of $P$ and PA averaged over Stokes parameters binned on 0.5 intervals.

In such a case some conclusions can be drawn about the magnetic field structure by comparing optical spectral curvature (spectral index) with spectral polarization. Examples of such an investigation can be found in articles by Holmes et al. (1984a) and Nordsieck (1976). The effect of the magnetic field geometry on the polarization has been considered by different authors (Nordsieck 1976; Björnsson 1982; Björnsson \& Blumenthal 1982). Björnsson \& Blumenthal have shown that for an aligned magnetic field the degree of polarization can be expressed as a function solely of spectral index, independent of the electron and magnetic field distribution. The degree of polarization should increase with spectral index only when the magnetic field alignment increases with field strength. However, a comparison of the theory and optical polarization observations and spectral index $\alpha$ in some quasars and BL Lac objects has shown that there are great deviations between observations and theoretical predictions (Nordsieck 1976).

To compare the relation between the variations of the degree of polarization of OJ 287 in 1994-1997 and the variations of the spectral index $\alpha$ we used the $R$-band data, since it has the best accuracy (Fig. 8).

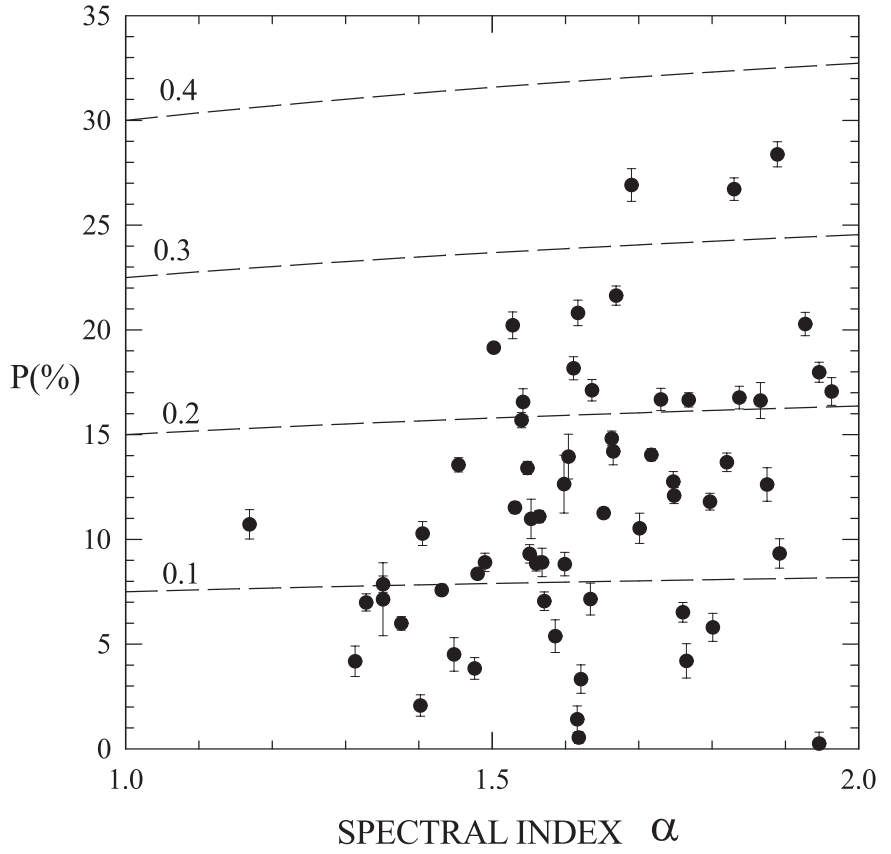

Fig. 8. Correlation between the variations of the degree of polarization $P$ of OJ 287 in $R$-band and the spectral index $\alpha$. Dashed lines mark the location of the theoretical degree of polarization for different alignments of the magnetic field in the jet. Figures near the lines indicate the degree of alignment (see text).

According to Björnsson \& Blumenthal (1982), the spectral dependence of the degree of polarizaton of the optically thin source can be evaluated by the formula

$P=c *(\alpha+1) /(\alpha+5 / 3)$,

where the coefficient $c$ represents the degree of the magnetic field alignment in the jet. The theoretical estimates of the degree of polarization are derived for values of the coefficient $c$ of $0.1,0.2,0.3$, and 0.4. A comparison of the observed and theoretically predicted degree of polarization reveals that the stronger the alignment of magnetic field the steeper the spectral energy distribution and the larger the degree of polarization. The largest polarization and the steepest spectral energy distribution are associated with the rather strong alignment of the magnetic field $(c>0.2)$.

We conclude from analysis of Figs. 4 and 7 that the values of the spectral index and degree of polarization gradually increase when the object brightens and reach their maxima at a moderate brightness level of about $14 \mathrm{~m} 5$. Further brightening results in weakening of the degree of polarization and flattening of the spectral index. Therefore, one expects the spectral index and degree of polarization to be correlated. In fact, despite the large amplitude polarization variations (more than 20\%) and the scatter of the spectral indices with brightness variations, such a correlation exists (Fig. 8). This correlation is not strong (the correlation coefficient is equal to $0.37 \pm 0.14$ ). However, it is seen that the polarization of OJ 287 becomes higher when the spectrum is steeper. 


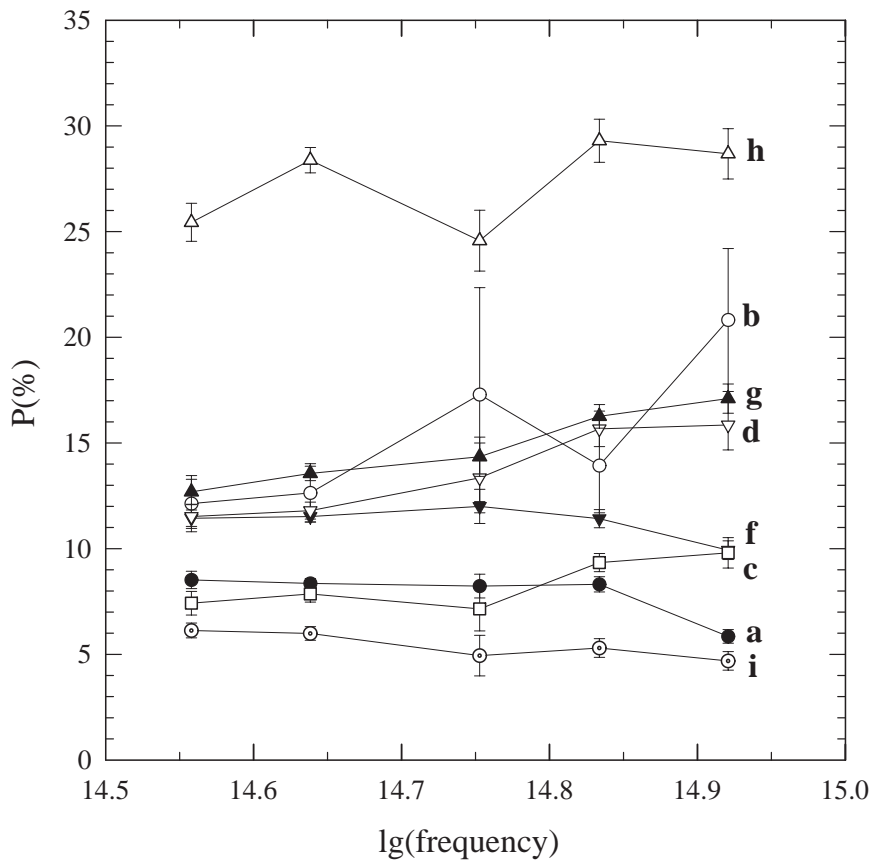

Fig. 9. Examples of the FDP of OJ 287: a) filled circles, JD 2449664; b) open circles, JD 2449797; c) filled triangles down, JD 2450070; d) open triangles down, JD 2450491; f) open squares, JD 2450134; g) filled triangles up, JD 2450135; h) open triangles up, JD 2450569; i) dotted circles, JD 2450155.

\subsection{Frequency dependence of polarization}

The frequency dependence of polarization $(F D P)$ and/or that of polarization position angle $(F D P A)$ is an important clue to the nature of the radiation in any object. A brief review of the proposed models has been presented by Takalo (Takalo 1994 and references therein). Since 1972 the shape of FDP and FDPA in OJ 287 has been studied by numerous authors with controversial conclusions (Strittmatter et al. 1972; Visvanathan 1973; Kikuchi et al. 1976; Puschell et al. 1983; Holmes et al. 1984a; Holmes et al. 1984b; Sitko et al. 1985; Kikuchi 1988; Ballard et al. 1990; Valtaoja et al. 1991; Sillanpää et al. 1991; Takalo et al. 1994; Smith 1996).

Some typical examples of the FDP and FDPA during our observations of OJ 287 are given in a paper by Efimov \& Shakhovskoy (1994), and in Figs. 9 and 10 at bright (maxima in 1994-1995 and 1995-1996), faint (before the maximum in 1994-1995 and between both maxima) and moderate brightness levels (1996-1997).

An inspection of all obtained $F D P$ and $F D P A$ shows a great variety of shapes, from rather flat to one with a rise of the degree of polarization toward shorter wavelength, as well as with a "hump" or depression in the visual region. The FDPA are usually independent or slightly dependent on wavelength. No evidence has been found that $F D P$ is related to the high polarization level.

The variety of forms in FDP and FDPA and their replacement may be considered as evidence for the

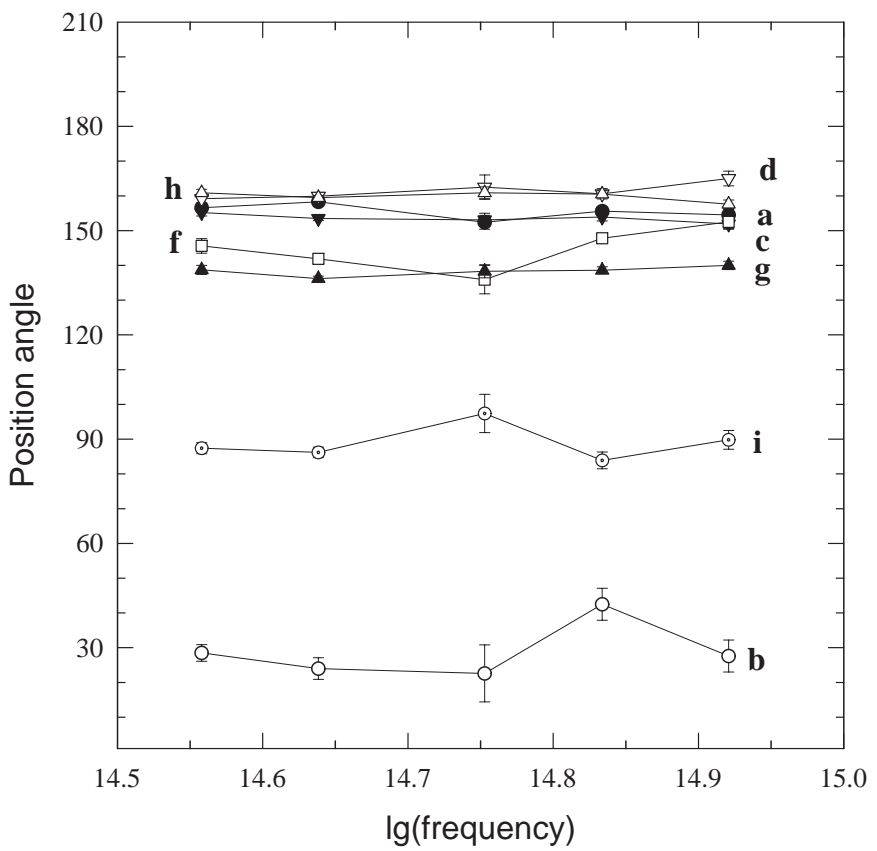

Fig. 10. Examples of the FDPA of OJ 287: a) filled circles, JD 2449664; b) open circles, JD 2449797; c) filled triangles down, JD 2450070; d) open triangles down, JD 2450491; f) open squares, JD 2450134; g) filled triangles up, JD 2450135; h) open triangles up, JD 2450569; i) dotted circles, JD 2450155.

appearance of several radiation components with different polarization parameters.

This is in agreement with Björnsson's (1985) note that strong FDP may be seen only when three requirements are simultaneously met: 1) well ordered magnetic field structure; 2) sharp high energy break or cut-off in the electron distribution; 3) non-uniform pitch-angle distribution skewed towards smaller values. It is not necessary that such conditions are always fulfilled. This could be the reason why observed $F D P$ are rare and transient.

\subsection{Relation between the polarization level and polarization angle}

According to Björnsson (1982), both beaming and twocomponent models predict that a large swing of polarization position angle is always associated with a state of low polarization. We have compared the variations of $P$ and PA in the $R$-band (Fig. 11). We see that the broad polarization maximum is centered at PA of about $170^{\circ}$. The significant scatter in the position angles at low-level polarization corresponds to larger uncertainties in the measurements when polarization is weak.

\subsection{Variation of the position angle with time}

One intriguing feature in OJ 287 is the large and rapid variations superimposed on a smooth, long-term change of the polarization angle. Rotation of the polarization plane in OJ 287 has been noted by different observers 


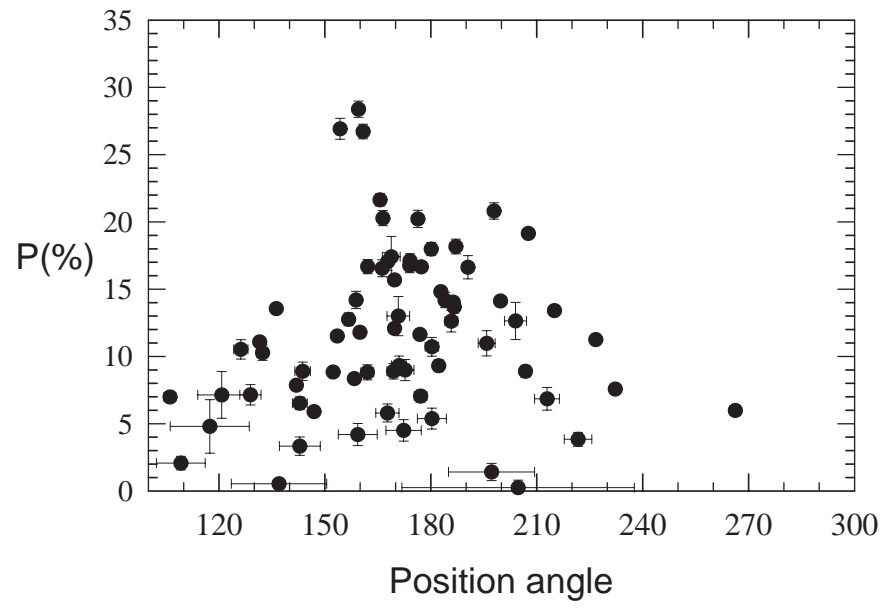

Fig. 11. Comparison of the variations of the polarization degree $P$ with the variations of the polarization position angle of OJ 287 in the $R$-band during 1994-1997.
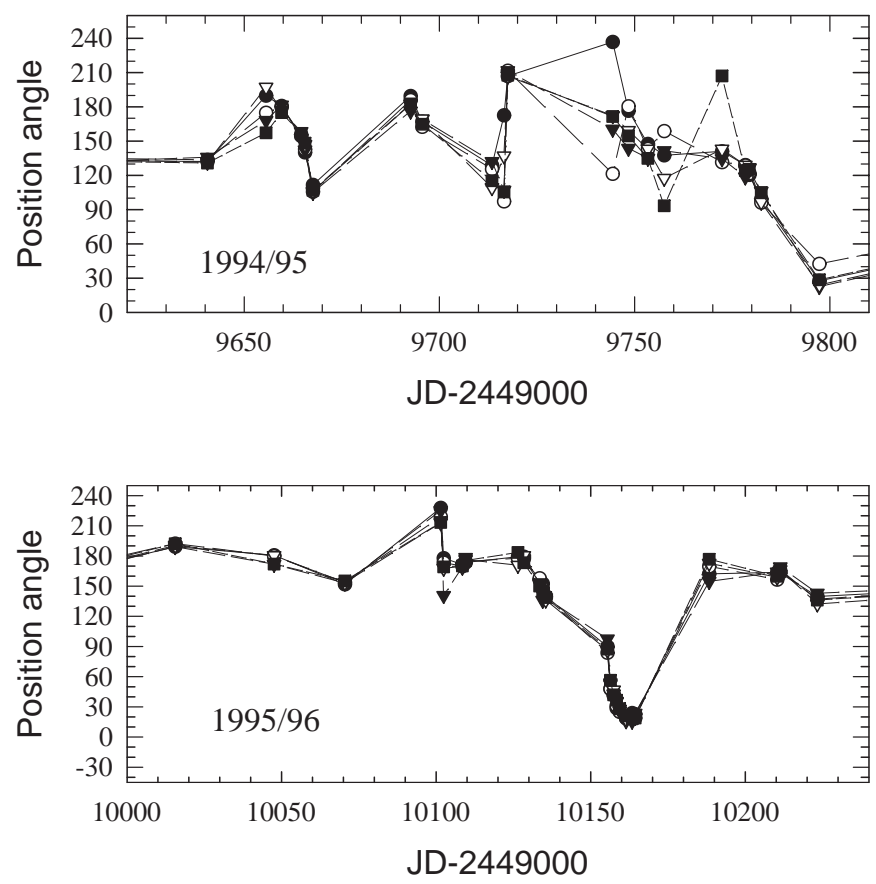

(e.g. Williams et al. 1972; Smith et al. 1987; Kikuchi et al. 1988; Kikuchi 1989; Sillanpää et al. 1991; Sillanpää et al. 1992; Takalo et al. 1994). A review of polarization position angle rotation in optical and radio domains between 1974-1991 was given by Sillanpää (1991).

Similar rotation of the position angle has also been found in some other blazars (PKS 0735+17, Efanov et al. 1981; BL Lac, Moore et al. 1987; Sillanpää et al. 1993).

A comparison of the variations of PA of OJ 287 is given in Fig. 12 for seasons 1994-1995 (upper panel) and 1995-1996 (middle panel), where different symbols relate to different wavebands. To eliminate the sharp jumps of $\mathrm{PA}$ from $0^{\circ}$ to $180^{\circ}$, due to the $180^{\circ}$ ambiguity in PA, the variations of $\mathrm{PA}$ in 1994-1997, were transferred into the interval $0^{\circ}-240^{\circ}$ by adding $180^{\circ}$ to all $\mathrm{PA}$ if $90^{\circ}<$ $\mathrm{PA}<180^{\circ}$. The results are shown in Fig. 12 . One can see that in all passbands the variations in PA, except for a few points, are almost identical. This allows us to calculate the weighted mean values for the position angles, MPA, using the data in all five bands for every night. These values will be used in further analysis.

The rotation of PA in 1994-1995 and 1995-1996 can be easily seen. The most remarkable systematic rotation of PA takes place during the time interval from January to March, 1996 (JD 2450100-2450170) when the PA rotates from $180^{\circ}$ to $0^{\circ}$ in about 70 days. In contrast to the previous data, the variations of PA in 1996-1997 are quite different: instead of the systematic rotation of PA, only fluctuations of PA between $145^{\circ}$ and $185^{\circ}$ are observed with a time scale of several days. Similar behaviour of PA was noted 10 years ago by Smith et al. (1987 and references therein) during the time of the 1983 outburst.

An example of the systematic rotation of MPA in $R$ band on February 12-March 20, 1996 (JD 24501262450163) is shown on the Stokes diagram in Fig. 13.

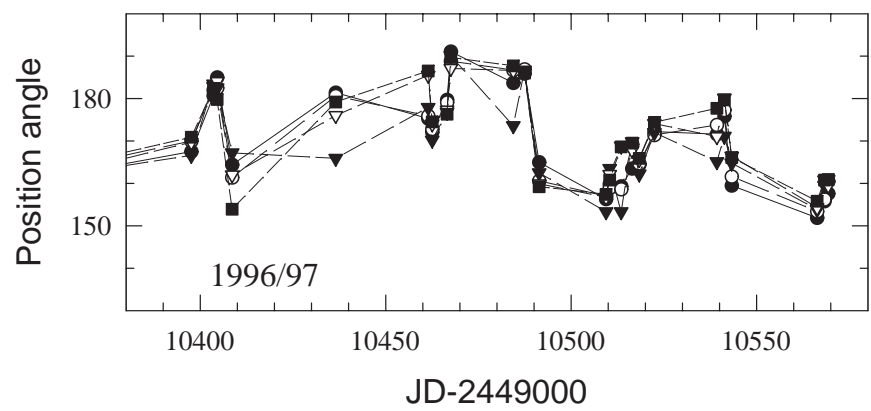

Fig. 12. Variations of the observed polarization position angles of OJ 287 in $U B V R I$ bands with time in JD during the monitoring campaign in 1994-1995 (upper panel), 1995-1996 (middle panel), and 1996-1997 (bottom panel). The used symbols are: filled circles for $U$, open circles for $B$, filled triangles down for $V$, open triangles down for $R$ and filled squares for I-band.

\section{Analysis of position angles variations}

If a gradual increase of the position angle MPA during the time interval $\Delta t$ is caused by a rotation of the polarization plane then the rotation period Per can be derived as $2 \pi / v$, where $v$ is the rotation rate in radians per day.

Time series analysis was applied to get the parameters $v$ and $\phi$ of the best fit to the MPA variations by a cosine function $\cos (v \Delta t+\phi)$, where $\phi$ is the initial phase of the period, $v$ is the rotation rate, and $\Delta t=\mathrm{JD}-\mathrm{JD}_{\mathrm{o}}$ is the time of the observations since the initial epoch $\mathrm{JD}_{\mathrm{o}}$.

Applying the periodogram analysis developed by Pelt (1992) to the MPA data in both 1994-1995 and 19951996 seasons, and accepting zero-point $\mathrm{JD}_{\mathrm{o}}=2449640.587$ (which is the starting date of our monitoring of OJ 287 in 1994-1995), we have found that the rate $v$ equals to $-4.92 /$ day, the duration of one $180^{\circ}$-circle is equal to 36.56 days, and the phase shift $\phi=209^{\circ} .6$. 


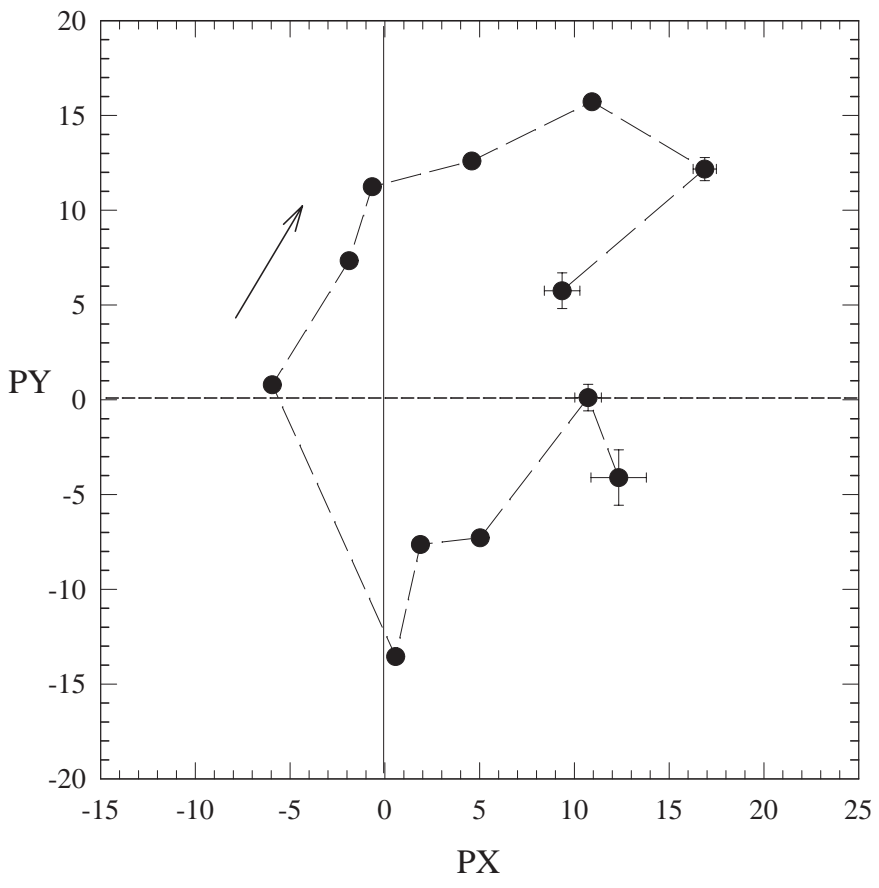

Fig. 13. Stokes diagram of the polarization of OJ 287 in the $R$-band in 1996. An arrow shows the direction of the polarization plane rotation in the time interval JD 2450126-2450163 (February 12-March 20, 1996).

The PA has the ambiguity $180^{\circ} * n$, where $n$ is an integer number, which may be calculated by using the formula

$n+p h=\left(\mathrm{JD}-\mathrm{JD}_{\mathrm{o}}\right) / P e r$,

where $p h$ is a phase (part of the period), JD and $\mathrm{JD}_{\mathrm{o}}$ are current and initial dates, and $P e r$ is the period of variation.

Knowing the cycle number $n$ for each observation and adding $180^{\circ} \times n$ to the observed position angle values, we may combine them to build a graph which shows the continuous rotation of MPA with time (Fig. 14, filled circles on upper panel). It is seen that all the data lie along a straight line. The slope of the regression line in Fig. 14 (solid line in the upper panel) corresponds to the rotation rate of MPA in 1994-1996 and is consistent with the value mentioned above.

Such a linear relation confirms the existence of the derived rotation rate of $\mathrm{PA}$ and allows us to interpret it as evidence for continuous rotation of the position angle of the source of polarized light.

The lower panel in Fig. 14 shows the comparison of the observed MPA (filled circles) with calculated angles (dashed line) reduced into interval $0^{\circ}-180^{\circ}$. It can be seen that in general the predicted angles agree with the behaviour of the observed position angles.

Some discrepancy may also be due to the observational errors and uncertainty in the period and phase determination as well as to our rather rough model, which cannot explain all observed features of the polarization variations.
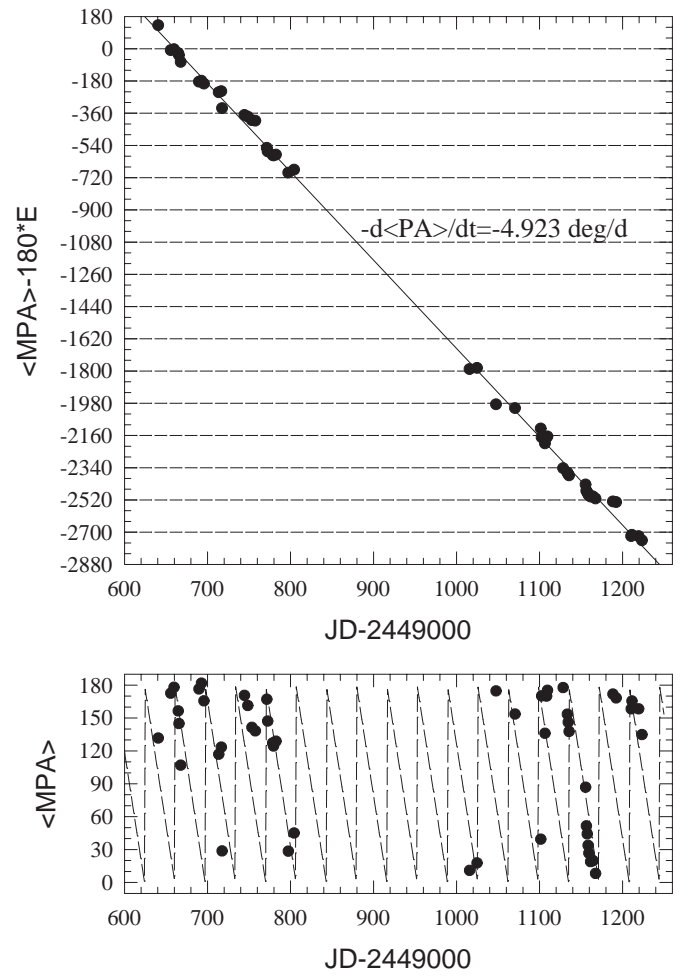

Fig. 14. The upper panel shows the progressive change of the mean position angle MPA in OJ 287 with time in JD (see text). The straight line is the regression line with the slope equal to the obtained rotation rate of MPA in 1994-1996. The lower panel shows the comparison between the variations of the mean position angles MPA (filled circles) with time in JD and the modeled change of PA with the rate $v$ equal to $-4.923 /$ day in the interval $0^{\circ}-180^{\circ}$ (dashed line).

\section{Discussion}

A number of models have been proposed to explain the variability of the polarization parameters: changes in the structure of the jet magnetic field (Shakhovskoy \& Efimov 1977), rotating clouds of relativistic electrons in the curved magnetic field (Hagen-Thorn 1980; Sillanpää et al. 1991), the "jet-shock" model by Königl \& Choudhuri (1985), developed for the optical region by Valtaoja et al. (1991 and references therein), flares in the accretion disk (Takalo et al. 1994 and references therein). The former was supported partly by the more or less correct predictions of the optical outbursts in OJ 287 on the basis of theoretical considerations by Lehto \& Valtonen (1996). In this model the rotation of PA can be caused by a polarized flaring component rotating in the accretion disk (Sillanpää et al. 1992). However, such a model cannot explain the very high degree of optical polarization that implies a synchrotron nature for the observed radiation. The main features of the observed variations in polarization parameters in OJ 287periodic or quasiperiodic variations of $\mathrm{PA}$ - also cannot be explained in the frame of a simple two-component model with constant and variable brightness sources with constant polarization parameters. 
A reasonable explanation can be given, based on the shock-jet model for the radio range (Valtaoja 1984; Marscher \& Gear 1985; Valtaoja et al. 1991), assuming that the jet in OJ 287, as well as in others blazars, is aligned with the line of sight (Blandford \& Königl 1979; Björnsson 1982). In this model the position of the polarization plane of the synchrotron source, which is responsible for the variable polarization, is connected with the transverse component of the magnetic field in the place of the jet where the perturbation (shock front or the clumps of relativistic particles) is located at the moment. In such a case the continuous progressive changes of the polarization plane orientation and cyclic variations of the observed PA are due to the helical structure of the magnetic field in the jet. Some theoretical arguments on the existence of helical magnetic field in jets have been presented by Ikhsanov \& Pustil'nik (1994) and by Vicente et al. (1996), based on VLBI observations. The possibility for such a structure based on optical observations was applied earlier by Kikuchi et al. (1988) to interpret the simultaneous position angle variation of OJ 287 in the optical and radio domains. Observational evidence for helical jet structure in blazars, in particular in OJ 287, has been presented by Villata \& Raiteri (1999 and references therein).

Our observations of OJ 287 during the outburst in 1994-1996 support the helical magnetic field structure in the blazar jets. Note, that there is no need for strict periodic variations in the position angles since both the spiral step as well as the apparent perturbation propagation rate may vary in the course of single outburst and different outbursts may occur in different places in the jet.

The polarization degree of the variable source may also vary due to properties of nonuniform magnetic field in the radiating volume and to the contribution from different radiation sources with various life-times to the total radiation.

Taking into consideration that several perturbations of different age and duration may exist simultaneously in various places of the jet, one can expect that the observed relations between intensity and polarization parameters may be very complicated. Such complicated behaviour of the observed parameters occurs very often in blazars.

\section{Conclusion}

The most important results from our photopolarimetric monitoring of blazar OJ 287 in 1994-1997 can be summarized as follows:

The analysis of the photopolarimetric data of OJ 287 reveals that no significant correlations between polarization and brightness are found during 1994-1997. The most plausible reason is the appearance and disappearance of polarized sources characterized by various polarization parameters, locations, and life times. The time scale of major variations in the degree of polarization may be as short as one or two days (e.g. data on polarization on February 20 and 21, 1996, JD 2450134 and 2450135, and on March 31 and April 2, 1997, JD 2450539 and 2450541).
The degree of polarization increased from a few percent in 1994 to 28\% in April 1997 (JD 2450550). Large night-to-night variations in the degree of polarization were observed. These variations look like a number of "polarization flashes" without any periodicity. This may be due to the existence of several sources with various life-times.

The analysis of our polarimetric observations of OJ 287 during the large outbursts in 1994-1996 has revealed cyclic variations of the polarization angle over $360^{\circ}$ with a period of about 73 days. These variations may be interpreted as continuous clockwise rotation of the polarization plane with a rate of about $5^{\circ}$ per day. From October 1994 until May 1996 the polarization plane of OJ 287 made 16 total $180^{\circ}$-slews. This is the first detection of such long-time (about three years) continuous rotation of the position angle of optical polarization in OJ 287. Such a rotation of the polarization plane may be explained in the framework of a model with perturbation (shock or clump of relativistic electrons) moving along a jet, pointed towards the observer, with a helical magnetic field structure.

Acknowledgements. We are grateful to V. Piirola for permission to use the photopolarimeter in these observations.

\section{References}

Ballard, K. R., Mead, A. R. G., Brand, P. W. J. L., \& Hough, J. H. 1990, MNRAS, 243, 640

Björnsson, C.-I. 1982, ApJ, 260, 855

Björnsson, C.-I. 1985, MNRAS, 216, 241

Björnsson, C.-I., \& Blumenthal, G. R. 1982, ApJ, 259, 805

Blake, G. M. 1970, ApL, 6, 201

Blandford, R. D., \& Königl, A. 1979, ApJ, 232, 34

Carini, M. T., Miller, H. R., Noble, J. C., et al. 1992, AJ, 104, 15

Dultzin-Hacyan, D., Takalo, L. 0., Benitez, E., et al. 1997, Rev. Mex. Astron. Astrofís., 33, 17

Efanov, V. A., Moiseev, I. G., Nesterov, N. S., et al. 1981, Izv. Krim. Astrofiz. Obs., 64, 91

Efimov, Yu. S., \& Shakhovskoy, N. M. 1994, in Workshop on Intensive Monitoring of OJ 287, Orilampi, Finland, 1720 June 1994, Ed. M. R., Kidger, \& L. O., Takalo, Tuorla Obs. Rep., 174, 28

Efimov, Yu. S., \& Shakhovskoy, N. M. 1996, in Proc. Workshop of Two Years of Intense Monitoring of OJ 287 and 3C 66A, Oxford (UK), 11-14 Sep. 1995, Two years of intensive monitoring of OJ 287 and 3C 66A, ed. L. O. Takalo, Tuorla Obs. Rep., 176, 32

Efimov, Yu. S., \& Shakhovskoy, N. M. 1998a, in Pubblicazioni Osservatorio Astronomico Universita di Perugia, 1998, OJ94 Annual Meeting 1997 An International Workshop on Multifrequency monitoring of Blazars, held in Perugia, Italy, 8-9th September, 1997, ed. G. Tosti, \& L. Takalo, vol. 3,30

Efimov, Yu. S., \& Shakhovskoy, N. M. 1998b, Blazar Data 1, No. 3

Fan, J. H., Adam, G., Xie, G. Z., et al. 1998, A\&AS, 133, 163

Fan, J. H. 2000, in press

Falomo, R., Scarpa, R., \& Bersanelli, M. 1994, ApJS, 93, 125 
Frölich, A., Goldsmith, S., \& Weistrop, D. 1974, MNRAS, 168, 417

Gear, W. K., Robson, E. I., \& Brown, L. M. J. 1986, Nature, 324,546

Hagen-Thorn, V. A. 1980, Ap\&SS, 73, 263

Hagen-Thorn, V.A., 1981, Transactions of the Astron. Observ. Leningrad State University, 36, 20

Holmes, P. A., Brand, P. W. J. L., Impey, C. D., et al. 1984a, MNRAS, 210, 961

Holmes, P. A., Brand, P. W. J. L., Impey, C. D., et al. 1984b, MNRAS, 211, 497

Hsu, J.-C., \& Breger, M. 1982, ApJ, 262, 732

Ikhsanov, N. R., \& Pustil'nik, L. A. 1994, A\&AS, 90, 959

Jia, G. B., Cen, X. F., Ma, H. Y., et al. 1998, A\&AS, 129,569

Kikuchi, S. 1988, PASJ, 40, 547

Kikuchi, S. 1989, Proc. Workshop on BL Lac Objects, Como, Italy, 20-23 Sept. 1988, ed. L. Maraschi, T. Maccacaro, \& M.-H. Ulrich, 131

Kikuchi, S., Mikami Y., Konno, M., \& Inoue, M. 1976, PASJ, 28,117

Kikuchi, S., Inoue, M., Mikami, Y., et al. 1988, A\&A, 190, L8

Korhonen, T., Piirola, V., \& Reiz, A. 1984, ESO Messenger 38, 20

Königl, A., \& Choudhuri, A. R. 1985, ApJ, 289, 188

Lehto, H., \& Valtonen, M. 1996, ApJ, 460, 207

Kümmel, M., Wagner, S. J., \& Heidt, J. 1994, AG Abstr. Ser., 10

Lyuty, V. M. 1976, Peremennye Zvezdy 20, 243

Marscher, A. P., \& Gear, W. K. 1985, ApJ, 298, 114

Moles, M., Garcia-Pelano, J., \& Masegosa, J. 1984, MNRAS, 211,621

Moore, R., Schmidt, G. D., \& West, S. C. 1987, ApJ, 314, 176

Nordsieck, K. H. 1976, ApJ, 209, 653

O'Dell, S. L., Puschell, J. J., Stein, W. A., et al. 1978, ApJ, 224, 22

Pelt, J. 1992, Irregular Spaced Data Analysis, User Manual, Helsinki (Helsinki University Press)

Piirola, V. 1973, A\&A, 27, 383

Piirola, V. 1988, in Polarized radiation of circumstellar origin, ed. G. V. Coyne, A. M. Magalhaes, A. F. J. Moffat, et al., Vatican Observatory - Vatican City State, 735

Pursimo, T., Takalo, L. O., Sillanpää, A., et al. 2000, A\&AS, 146,141

Puschell, J. J., Jones, T. W., Phillips, A. C., et al. 1983, ApJ, 265,625

Rieke, G. H., \& Kinman, T. D. 1974, ApJ, 192, L115
Serkowski, K. 1974, in Planets, Stars and Nebulae, studied with Photopolarimetry, ed. T. Gehrels (University of Arizona Press, Tucson), 135

Shakhovskoy, N. M., \& Efimov, Yu. S. 1977, Izv. Krim. astrofiz. obs., 56,39

Sillanpää, A. 1991, A\&A, 247, 11

Sillanpää, A., Haarala, S., Valtonen, et al. 1988, ApJ, 325, 628

Sillanpää, A., Takalo, L. O., Kikuchi, S., et al. 1991, AJ, 101, 2017

Sillanpää, A., Takalo, L. O., Nilsson, K., et al. 1992, A\&A, 254, L33

Sillanpää, A., Takalo, L. O., Nilsson, K., et al. 1993, Ap\&SS, 206,55

Sillanpää, A., Takalo, L. O., Lehto, H., et al. 1996, A\&A, 305, L17

Sitko, M. L., Schmidt, G. D., \& Stein, W. A., et al. 1985, ApJSS, 59, 323

Smith, P. S. 1996, ASP Conf. Ser., 110, 135

Smith, P. S., Balonek, T. J., Heckert, P. A., et al. 1985, AJ, 90, 1184

Smith, P. S., Balonek, T. J., Heckert, P. A., et al. 1986, ApJ, 305,484

Smith, P. S., Balonek, T. J., Elston, R., \& Heckert, P. A. 1987, ApJS, 64, 459

Strittmatter, P. A., Serkowski, K., Carswell, R., et al. 1972, ApJ, 175, L7

Takalo, L. O. 1994, Vistas in Astronomy, 38, 77

Takalo, L. O., \& Sillanpää, A. 1989, A\&A, 218, 45

Takalo, L. O., Sillanpää, A., Nilsson, K., et al. 1994, A\&AS, 104,115

Tosti, G., Fiorucci, M., Luciani, M., et al. 1998, A\&A, 339, 41

Valtaoja, E. 1984, Ap\&SS, 100, 227

Valtaoja, L., Valtaoja, E., Shakhovskoy, N. M., et al. 1991, AJ, 101,78

Valtaoja, L., Karttunen, H., Efimov, Yu., et al. 1993, A\&A, 278,371

Vicente, L., Charlot, P., \& Sol, H. 1996, A\&A, 312, 727

Villata, M., \& Raiteri, C. M., in BL Lac phenomenon, Proc. of the meeting held at Turku, Finland, 22-26 June 1998, ed. L. O. Takalo, \& A. Sillanpää, PASP, 159, 48

Villata, M., \& Raiteri, C. M. 1999, A\&A, 347, 30

Visvanathan, N. 1973, ApJ, 185, 145

Williams, W. L., Rich, A., Kupferman, P. N., et al. 1972, ApJ, 174, L63

Worrall, D. M., Puschell, J. J., Jones, B., et al. 1982, ApJ, 261, 403 